\title{
LncRNA AW112010 Promotes Mitochondrial Biogenesis and Hair Cell Survival: Implications for Age-Related Hearing Loss
}

\author{
Zhongwu Su, ${ }^{1,2}$ Hao Xiong, 1,2 Jiaqi Pang, ${ }^{1,2}$ Hanqing Lin,, ${ }^{1,2}$ Lan Lai, ${ }^{1,2}$ Huasong Zhang, ${ }^{1,2}$ \\ Weijian Zhang, ${ }^{1,2}$ and Yiqing Zheng iD ${ }^{1,2}$ \\ ${ }^{1}$ Department of Otolaryngology, Sun Yat-sen Memorial Hospital, Sun Yat-sen University, Guangzhou, China \\ ${ }^{2}$ Institute of Hearing and Speech-Language Science, Sun Yat-sen University, Guangzhou, China \\ Correspondence should be addressed to Yiqing Zheng; zhengyiq@mail.sysu.edu.cn
}

Received 6 March 2019; Revised 12 June 2019; Accepted 19 August 2019; Published 27 October 2019

Academic Editor: Mario Zoratti

Copyright (C) 2019 Zhongwu Su et al. This is an open access article distributed under the Creative Commons Attribution License, which permits unrestricted use, distribution, and reproduction in any medium, provided the original work is properly cited.

\begin{abstract}
Long noncoding RNA (lncRNA) disorder has been found in many kinds of age-associated diseases. However, the role of lncRNA in the development of age-related hearing loss (AHL) is still largely unknown. This study sought to uncover AHL-associated lncRNAs and the function. RNA-sequencing was conducted to profile lncRNA expression in the cochlea of an early-onset AHL mouse model. RT-qPCR assay was used to validate the expression pattern of lncRNAs. ATP assay, JC-1 assay, mitochondrial probe staining, CCK-8 assay, Western blot, and immunocytochemistry were performed to detect the effects of lncRNA AW112010 in HEI-OC1 cells and the mouse cochlea. We identified 88 significantly upregulated lncRNAs and 46 significantly downregulated lncRNAs in the cochlea of aged C57BL/6 mice. We focused on the significantly upregulated AW112010. Silencing of AW112010 decreased the ATP level, mitochondrial membrane potential, and cell viability and increased mitochondrial ROS generation under oxidative stress in HEI-OC1 cells. AW112010 overexpression promoted cell survival in HEI-OC1 cells. AW112010 knockdown reduced mitochondrial mass and impaired mitochondrial biogenesis in HEI-OC1 cells. Activation of mitochondrial biogenesis by resveratrol and STR1720 promoted cell survival. The mitochondrial biogenesis process was activated in the cochlea of aged mice. Moreover, AW112010 regulated AMPK signaling in HEI-OC1 cells. Transcription factor Arid5b elevated in the aged cochlea and induced AW112010 expression and mitochondrial biogenesis in HEI-OC1 cells. Taken together, lncRNAs are dysregulated with aging in the cochlea of C57BL/6 mice. The Arid5b/AW112010 signaling was induced in the aged mouse cochlea and positively modulated the mitochondrial biogenesis to maintain mitochondrial function.
\end{abstract}

\section{Introduction}

Age-related hearing loss (AHL), also known as presbycusis, is the most common sensory disorder in old people, affecting about $20-40 \%$ of people by age 65 years and older $[1,2]$. It significantly affects the daily communication of the old people. Intrinsic factors (e.g., genetic predisposition) and extrinsic factors (e.g., noise exposure) together result in the occurrence of AHL during aging [1]. The irreversible loss of cochlear hair cells is one of the major pathological changes of AHL [3-5]. Oxidative stress, mitochondrial DNA mutations/deletions, decreased autophagy, and microRNA disorder account for the death of hair cells [1, $2,4,5]$. However, the mechanism of hair cell loss is still not fully understood.
Long noncoding RNAs (lncRNAs) are a class of RNA that are longer than 200 nucleotides and do not have the potential to code proteins $[6,7]$. Recent studies have revealed that $\operatorname{lncRNAs}$ play significant roles in the regulation of gene expression and participate in multiple biological processes, including cell growth, apoptosis, and differentiation $[6,7]$. LncRNA disorder has been found in many kinds of diseases, such as cancer and cardiovascular and neurodegenerative diseases [8]. LncRNAs have also been reported to be involved in the pathophysiological processes in the ear. A recent study revealed differential IncRNA profile between two developmental stages of the mouse inner ear sensory epithelium of the cochlea and vestibule, suggesting a possible role for lncRNAs in regulating hearing and balance [9]. A study in a Chinese population unveiled that IncRNA HOTAIR 
polymorphism was associated with the occurrence of noiseinduced hearing loss [10]. Nevertheless, it remains largely unknown whether lncRNAs participate in the development of AHL.

Mitochondria have a vital role in maintaining cellular homeostasis $[11,12]$. Growing evidence suggests that mitochondrial dysfunction participates in aging diseases, such as diabetes, neurodegenerative disease [12], and AHL [1]. Mitochondrial biogenesis is a tightly regulated process to generate new mitochondria and plays an important role in maintaining normal mitochondrial function $[11,12]$. The progress is orchestrated by a series of transcription factors, such as peroxisome proliferator-activated receptor gamma coactivator 1-alpha (PGC-1 $\alpha$ ) and mitochondrial transcription factor A (TFAM), and is under the control of AMP-activated protein kinase (AMPK), the mammalian target of rapamycin (mTOR), and insulin-like signaling (ILS) pathways $[11,12]$. As important gene regulation factors, lncRNAs also modulate the expression of genes involved in the mitochondrial biogenesis process [13].

In the present study, we hypothesized that lncRNAs are dysregulated in AHL and play harmful or protective roles in the survival of cochlear hair cells. To address this, we firstly profiled the IncRNA expression in the cochlear tissues of an early-onset AHL mouse model and identified lncRNA AW112010 significantly upregulated in the aged cochlea. Next, we analyzed the effect of AW112010 on mitochondrial function and biogenesis and on the activity of AMPK signaling. Finally, the upstream regulator of AW112010 was explored.

\section{Materials and Methods}

2.1. Animals. 50 C57BL/6 mice (Laboratory Animal Center, Sun Yat-sen University, Guangzhou, China) were divided into 2 groups: a "young” group (4 weeks old, 25 subjects) and an "old" group (12 months old, 25 subjects). All experiments were performed according to the protocols approved by the Animal Research Center, Sun Yat-sen University.

2.2. Auditory Brainstem Response. Auditory brainstem response (ABR) tests were conducted using a Tucker-Davis Technology (TDT) System III (Alachua, FL, USA) as previously described $[4,5]$. Briefly, mice were subcutaneously inserted with subdermal needle electrodes at the vertex (active) and below the left ear (reference) and the right ear (ground) after being anesthetized. ABR tests were measured at 8,16 , and $32 \mathrm{kHz}$. The average response to 1024 stimuli was obtained through reducing the sound intensity at $5 \mathrm{~dB}$ intervals near the threshold, which was defined as the lowest stimulus level where a positive wave was evident.

2.3. Tissue Preparation. After ABR tests, the deeply anesthetized mice were decapitated. The cochlea were obtained and fixed with $4 \%$ paraformaldehyde at $4^{\circ} \mathrm{C}$ overnight and decalcified in $4 \%$ sodium ethylenediaminetetraacetic acid for two days. For RNA and protein preparation, cochlear tissues were dissected and snap frozen in liquid nitrogen and stored at $-80^{\circ} \mathrm{C}$.
2.4. RNA Extraction and Sequencing. Total RNA was extracted using a TRIzol reagent (Invitrogen, USA) following the manufacturer's instructions. For RNA-sequencing, cochlear tissues from 3 mice were collected and mixed as one sample. RNA purity, concentration, and integrity were measured using a NanoPhotometer spectrophotometer (IMPLEN, USA), Qubit 2.0 Fluorometer (Life Technologies, USA), and Bioanalyzer 2100 system (Agilent Technologies, USA), respectively. LncRNA sequencing libraries were generated using a NEBNext Ultra ${ }^{\mathrm{TM}}$ Directional RNA Library Prep Kit for Illumina (NEB, USA) according to the manufacturer's recommendations. The libraries were sequenced on an Illumina Hiseq 4000 platform, and $150 \mathrm{bp}$ paired-end reads were generated.

2.5. Differential Expression Analysis. Data filtering was performed, and clean data were obtained. The clear reads were then aligned to the reference mouse genome (GRCm38). The mapped reads of each sample were then assembled by Cufflinks (v2.1.1) in a reference-based approach. Cuffdiff (v2.1.1) was used to calculate FPKMs (expected number of Fragments Per Kilobase of transcript sequence per Million base pairs sequenced) of lncRNAs in each sample and to analyze the differential expression. Transcripts with $p$-adjust $<0.05$ were assigned as differentially expressed. Volcano plot and heat map were generated based on the differentially expressed transcripts.

2.6. Cell Culture. Cochlear HEI-OC1 cells (kindly provided by F. Kalinec at the House Ear Institute, Los Angeles, CA, USA) were cultured in Dulbecco's modified Eagle's medium (Gibco, USA), supplemented with $10 \%$ fetal bovine serum (Gibco) at $33^{\circ} \mathrm{C}$ under $10 \% \mathrm{CO}_{2}$ condition.

2.7. Cell Transfection. The AW112010 Smart Silencer (Ruibo, Guangzhou, China), a commercial mixture of siRNA and ASO (antisense oligonucleotides), was used to knock down the expression of AW112010 in HEI-OC1 cell. The pcDNA3.1AW112010 plasmid (IGE Biotechnology, Guangzhou, China) was used to express AW112010 in HEI-OC1 cell. Arid5b siRNA (GenePharma, Shanghai, China) was applied to knock down the expression of Arid5b in HEI-OC1 cell. Cell transfections were performed using a Lipofectamine RNAiMAX Transfection Reagent (Life Technologies, USA) or FuGENE ${ }^{\circledR}$ HD Transfection Reagent (Promega, USA) following the manufacturer's instructions.

2.8. Quantitative Reverse Transcription-Polymerase Chain Reaction (RT-qPCR). According to the manufacturer's protocols, $500 \mathrm{ng}$ total RNA was reverse-transcribed using PrimeScript RT Master Mix (Takara, Japan). A qPCR assay was performed using TB Green Premix Ex Taq II (Takara) on a Roche LightCycler 96 real-time PCR system (Roche, Switzerland). Primers for AW112010, Gm44593, 1700030C10Rik, 5330434G04Rik, Rian, and H19 were purchased from Ruibo (Guangzhou, China). Other primer sequences used were as follows: Arid5b: forward: $5^{\prime}$ -GTGATGAGTTCGCGCCAAATC-3' , reverse: $5^{\prime}$-GCTG ATAACTTTACCGTCACAGT-3' and glyceraldehyde-3- 
phosphate dehydrogenase (GAPDH): forward: $5^{\prime}$-GGTC ATCCATGACAACTTTGG- ${ }^{\prime}$, reverse: $5^{\prime}$-GGCCATCAC GCCACAG- $3^{\prime}$. The expression levels of genes were normalized to GAPDH.

2.9. Western Blot Analysis. Western blot was conducted as previously described $[4,5]$. Briefly, proteins were extracted from cells and cochlea tissues using radioimmunoprecipitation assay lysis buffer (Thermo plus, USA). Protein samples $(20 \mu \mathrm{g})$ were resolved on $10 \%$ SDSpolyacrylamide gel and transferred onto polyvinylidene fluoride membranes (Millipore, USA). After blocking with $5 \%$ nonfat milk, the membranes were incubated with antiPGC- $1 \alpha$ (1:1000, Abcam, USA), anti-TFAM ( $1: 1000$, Abcam), anti-p-AMPK (1:1000, Cell Signaling Technology, USA), anti-AMPK $(1: 1000$, Cell Signaling Technology, USA), and anti-Arid5b $\left(1: 500\right.$, Abgent, USA) at $4^{\circ} \mathrm{C}$ overnight, followed with secondary antibodies $(1: 10000)$ at room temperature for $1 \mathrm{~h}$. Then, the immunoreactive bands were detected using enhanced chemiluminescence (Millipore, USA). Band intensities were analyzed using NIH ImageJ. $\beta$ Actin was applied as loading and internal control.

2.10. Immunocytochemistry. After decalcification, cochlear tissues were immersed in 3\% Triton X-100 solution for $30 \mathrm{~min}$ at room temperature. After washing with PBS, the tissues were blocked with $10 \%$ goat serum for $30 \mathrm{~min}$ at room temperature. Then, cochlear tissues were incubated with antibody anti-PGC- $1 \alpha(1: 100, \mathrm{Abcam})$ at $4^{\circ} \mathrm{C}$ for $24 \mathrm{~h}$. After washing with PBS, cochlear tissues were incubated with Alexa Fluor 594 secondary antibody $(1: 200$, Invitrogen) at $4^{\circ} \mathrm{C}$ overnight in darkness. Following PBS washes, the tissues were then incubated with Alexa Fluor 488 phalloidin (1:100, Invitrogen) at room temperature for $1 \mathrm{~h}$. Then, the tissues were washed and counterstained with DAPI for 30 minutes and imaged with Zeiss LSM 710 confocal microscopy (Zeiss, Germany). Images were then analyzed using NIH ImageJ software.

2.11. Cell Viability Assay. Cells were treated with $1 \mathrm{mM} \mathrm{H}_{2} \mathrm{O}_{2}$ (Sigma-Aldrich, USA) for $2 \mathrm{~h}$. To activate mitochondrial biogenesis, cells were preincubated with $5 \mu \mathrm{M}$ resveratrol (Sigma-Aldrich) or $0.5 \mu \mathrm{M}$ SRT1720 (Selleck, USA) for $24 \mathrm{~h}$. Cell viability was detected using CCK-8 kits (DOJINDO, Japan) according to the manufacturer's protocols. Briefly, cells were plated into a 96-well plate. At the indicated time after treatments, $10 \mu \mathrm{l} \mathrm{CCK-8} \mathrm{solution} \mathrm{was} \mathrm{added}$ into the wells. Cells were then incubated at $37^{\circ} \mathrm{C}$ in an incubator for $1 \mathrm{~h}$. Then, a microplate reader (Labsystems Dragon, Finland) was used to measure the absorbance at $450 \mathrm{~nm}$.

2.12. ATP Assay. An ATP assay was performed using an ATP Assay Kit (Beyotime, Shanghai, China) following the manufacturer's instructions. Briefly, cells were homogenized with lysis buffer and centrifuged at $12000 \times \mathrm{g}$ for $5 \mathrm{~min}$ at $4^{\circ} \mathrm{C}$. The ATP detection reagent was diluted with dilution buffer and added into a 96-well plate. Then, samples were added into wells and mixed with detection solution. Chemiluminescence of samples and standards were measured with

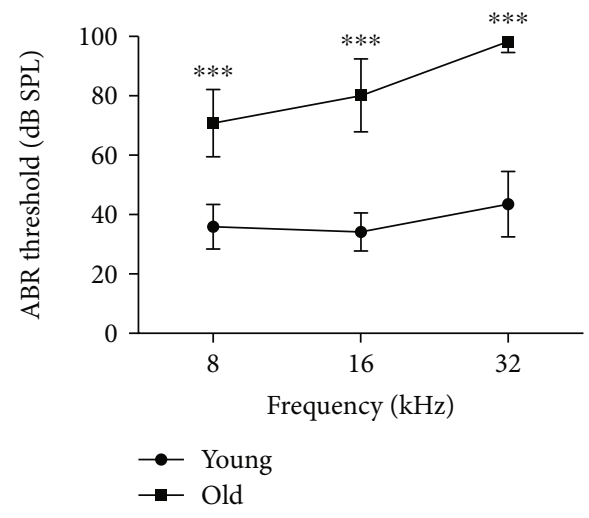

FIGURE 1: Elevated auditory brainstem response thresholds in aging C57BL/6 mice. Increased auditory brainstem response thresholds were observed in 12-month-old C57BL/6 mice at 8,16 , and $32 \mathrm{kHz} .{ }^{* * *} p<0.001$ relative to 4 -week-old C57BL/6 mice, $n=25$.

a SpectraMax M5 microplate reader (Molecular Devices, USA). The levels of ATP were calculated based on the standard curve and normalized to the protein content.

2.13. Mitochondrial Staining. Mitochondrial staining was conducted with the mitochondrial probe MitoTracker Red CMXRos (Yeasen, Shanghai, China) according to the manufacturer's protocols. Briefly, cells were incubated with CMXRos $(500 \mathrm{nM})$ at $37^{\circ} \mathrm{C}$ in an incubator for $30 \mathrm{~min}$. After washing with PBS, the cells were counterstained with DAPI for 10 minutes and imaged with an Olympus BX63 microscope (Olympus, Japan).

2.14. Mitochondrial Reactive Oxygen Species (ROS) Detection. Mitochondrial ROS levels were measured by staining with MitoTracker Red CM-H2XRos (Invitrogen) following the manufacturer's instructions. Briefly, cells were incubated with $\mathrm{CM}-\mathrm{H} 2 \mathrm{XR}$ os $(500 \mathrm{nM})$ at $37^{\circ} \mathrm{C}$ in an incubator for 30 min. After washing with PBS, the cells were counterstained with DAPI for 10 minutes and imaged with an Olympus BX63 microscope (Olympus).

2.15. Mitochondrial Membrane Potential (MMP) Assay. A JC-1 Kit (GeneCopoeia, USA) was used to measure mitochondrial membrane potential following the manufacturer's instructions. Briefly, cells were incubated with a diluted JC-1 reagent $(1: 100)$ at $37^{\circ} \mathrm{C}$ in an incubator for $30 \mathrm{~min}$. Then, cells were analyzed by flow cytometry with FACSVerse (BD Biosciences, USA).

2.16. Mitochondrial DNA (mtDNA) Content Analysis. Total genomic DNA was extracted from cells using a Universal Genomic DNA Extraction Kit (Takara) according to the manufacturer's protocols. The mtDNA levels were quantified by qPCR on a Roche LightCycler 96 (Roche) using D-loop primers (forward: 5'-GGTTCTTACTTCAGGG CCATCA-3', reverse: $5^{\prime}$-GATTAGACCCGTTACCATC GAGAT- ${ }^{\prime}$ ). Nuclear gene beta2-microglobulin (B2M) primers (forward: $5^{\prime}$-ATGGGAAGCCGAACATACTG-3', 


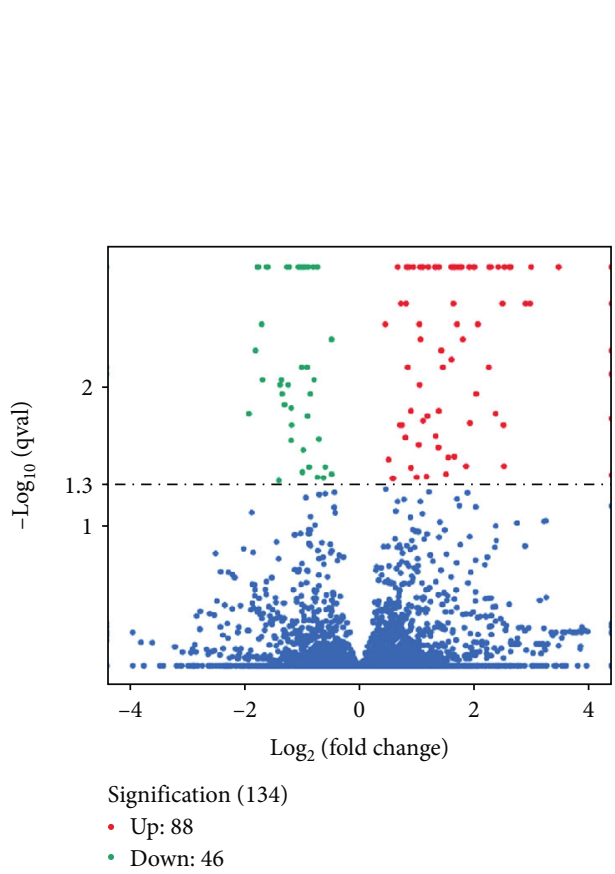

(a)

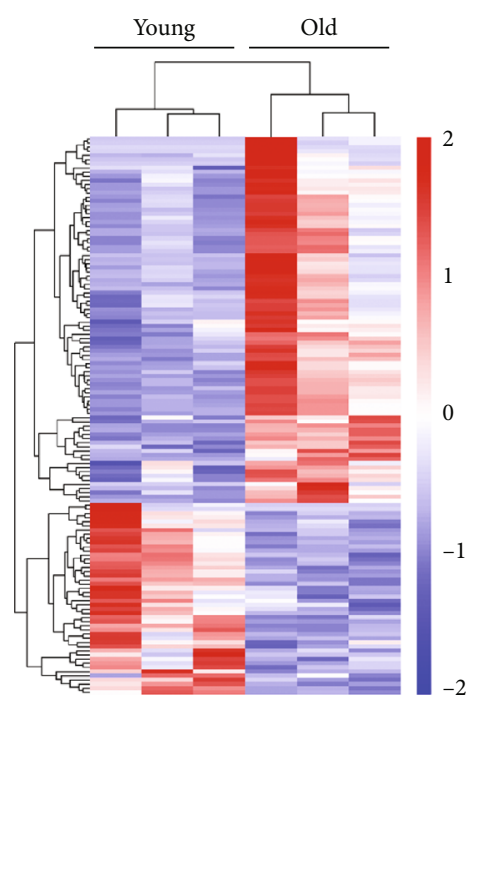

(b)

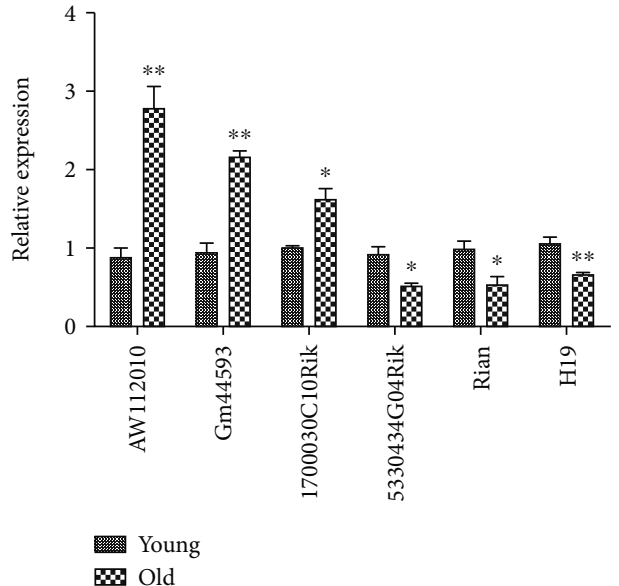

(c)

FIgURE 2: Differentially expressed lncRNAs in the cochlea of aged C57BL/6 mice. (a) Volcano plot showing comparative cochlea lncRNA gene expression in 4-week (young)- and 12-month (old)-old C57BL/6 mice $(n=3)$. Red dots indicated the significantly increased transcripts (88), and blue dots represented the significantly decreased transcripts $(46)$ in old mice ( $p$-adjust $<0.05)$. (b) Heat map showing hierarchical clustering of differentially expressed lncRNAs. Red indicated high relative expression, and blue represented low relative expression. (c) RT-qPCR analysis validation of 6 differentially expressed lncRNAs. ${ }^{*} p<0.05,{ }^{* *} p<0.01$.

reverse: $5^{\prime}$-CAGTCTCAGTGGGGGTGAAT-3') were used as a nuclear control.

2.17. Statistical Analysis. All experiments were independently repeated at least 3 times. Data were presented as the mean \pm SEM and analyzed with SPSS software and GraphPad Prism 5 software. Student's $t$-test and one-way ANOVA were used for statistical analysis. Values of $p<0.05$ were considered significant.

\section{Results}

3.1. Progressive Hearing Loss with Aging in C57BL/6 Mice. Auditory brainstem response (ABR) tests were performed to examine hearing function in $\mathrm{C} 57 \mathrm{BL} / 6$ mice. The average thresholds from old mice $(71 \pm 11 \mathrm{~dB}$ at $8 \mathrm{kHz}, 80 \pm 13 \mathrm{~dB}$ at $16 \mathrm{kHz}$, and $99 \pm 4 \mathrm{~dB}$ at $32 \mathrm{kHz}$ ) were significantly elevated compared with those from young mice $(36 \pm 7 \mathrm{~dB}$ at $8 \mathrm{kHz}, 34 \pm 6 \mathrm{~dB}$ at $16 \mathrm{kHz}$, and $43 \pm 11 \mathrm{~dB}$ at $32 \mathrm{kHz}$ ) (Figure 1). These data indicate that C57BL/6 mice developed early-onset AHL.

3.2. Differential Expression Profile of LncRNA in the Cochlea of Aged C57BL/6 Mice. To identify AHL-related lncRNAs, cochlear tissues from young and old C57BL/6 mice were collected for RNA-sequencing. Transcriptome analysis identified 88 upregulated and 46 downregulated lncRNAs in old mice compared to the young mice ( $p$-adjust $<0.05$, Figure 2(a)). Volcano plot (Figure 2(a)) and heat map (Figure 2(b)) were generated using the differentially expressed lncRNAs. To confirm the sequencing results, three upregulated and three downregulated lncRNAs were randomly selected for validation using RT-qPCR. The expression patterns of all transcripts were consistent with the RNA-sequencing analysis (Figure 2(c)). These results suggest that lncRNA disorder was accompanied with the development of AHL in C57BL/6 mice.

3.3. AW112010 Promotes Mitochondrial Function and Cell Survival in HEI-OC1 Cells. Among these dysregulated lncRNAs, we focused on the significantly upregulated gene AW112010, which has been reported to be induced following virus infection in microglia and astrocytes [14]. Firstly, we examined the expression pattern of AW112010 in HEI-OC1 cells under $\mathrm{H}_{2} \mathrm{O}_{2}$ treatment and serum deprivation to mimic the condition of oxidative stress and energy shortage in vivo. As observed in the aged cochlea sample, the expression of AW112010 was markedly induced in response to $\mathrm{H}_{2} \mathrm{O}_{2}$ $(1 \mathrm{mM})$ stimulation $(p<0.05$, Figure $3(\mathrm{a}))$ and serum deprivation $(p<0.05$, Figure $3(\mathrm{~b}))$. Further, Compound $C$, a specific AMPK inhibitor, was used to block the energysensitive AMPK signaling. The RT-qPCR assay showed that Compound $C$ decreased the expression of AW112010 $(p<0.05$, Figure $3(\mathrm{c}))$. These results indicate that 


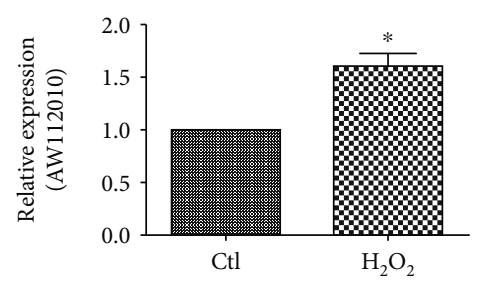

(a)

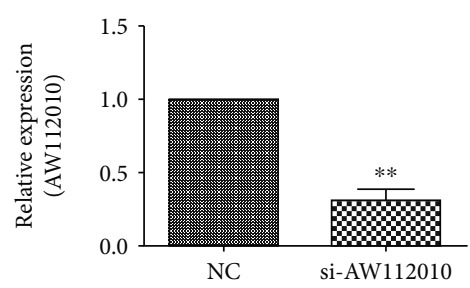

(d)

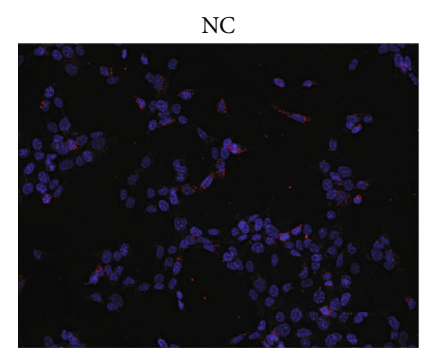

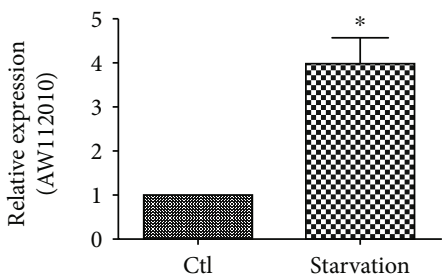

(b)

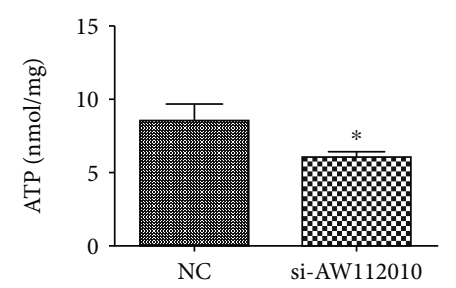

(e)

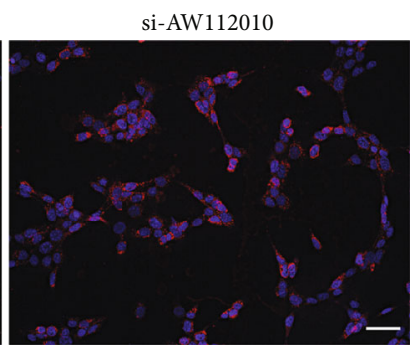

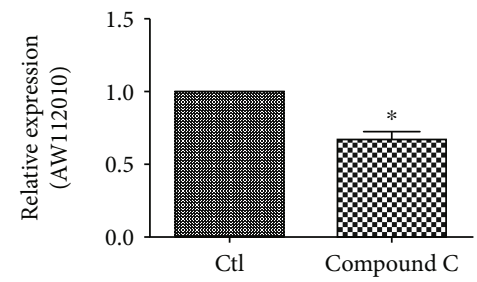

(c)

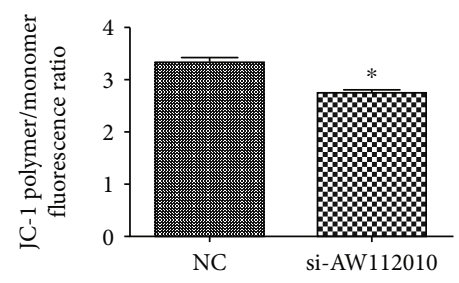

(f)

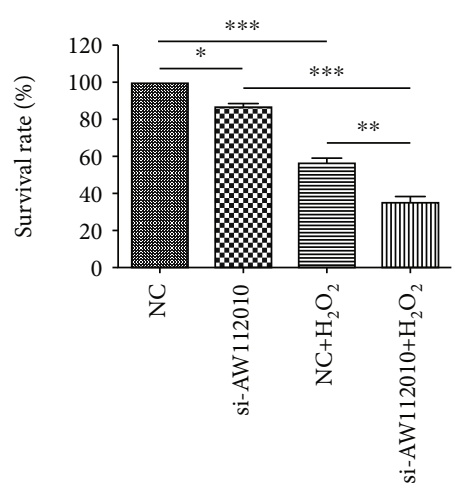

(h) (g)

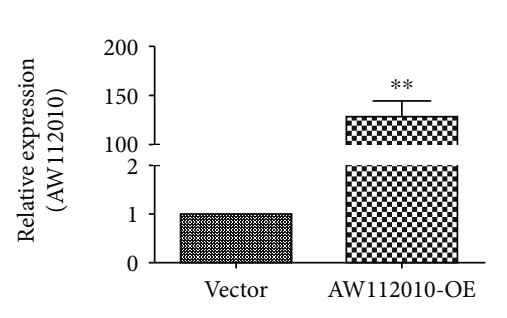

(i)

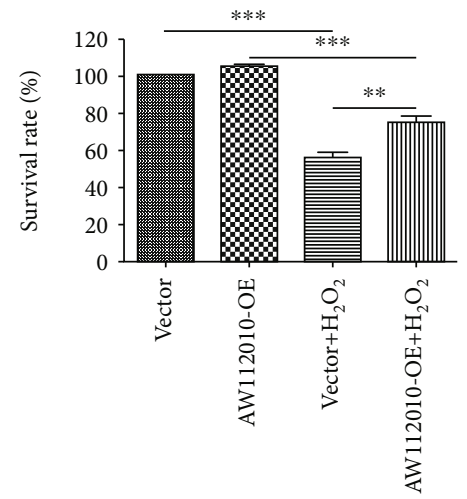

(j)

FIGURE 3: LncRNA AW112010 promoted mitochondrial function and cell survival in HEI-OC1 cells. (a) HEI-OC1 cells were treated with $1 \mathrm{mM} \mathrm{H} \mathrm{O}_{2}$ for $6 \mathrm{~h}$. RT-qPCR analysis showed that $\mathrm{H}_{2} \mathrm{O}_{2}$ treatment increased AW112010 expression in HEI-OC1 cells. (b) Serum deprivation elevated AW112010 expression in HEI-OC1 cells. (c) HEI-OC1 cells were incubated with $5 \mu \mathrm{M}$ Compound C for 24 h. RTqPCR analysis showed that Compound C decreased AW112010 expression in HEI-OC1 cells. (d) RT-qPCR analysis validation of AW112010 knockdown (si-AW112010) by Smart Silencer in HEI-OC1 cells. (e) ATP assay showed reduced ATP levels in AW112010 knockdown cells compared to negative control cells. (f) JC-1 assay revealed a decreased JC-1 polymer/monomer fluorescence ratio in AW112010 knockdown cells. (g) Mitochondrial ROS was measured using CM-H2XRos probes. Immunocytochemical analysis showed elevated red fluorescence in AW112010 knockdown cells under $1 \mathrm{mM} \mathrm{H}_{2} \mathrm{O}_{2}$ stimulation. Scale bar: $50 \mu \mathrm{m}$. (h) CCK-8 assay revealed decreased cell viability in AW112010 knockdown cells in the presence or absence of $1 \mathrm{mM} \mathrm{H}_{2} \mathrm{O}_{2}$ treatment compared to negative control cells. (i) RT-qPCR analysis validation of AW112010 overexpression (AW112010-OE) in HEI-OC1 cells. (j) CCK-8 assay showed increased cell viability in AW112010 overexpression cells under $\mathrm{H}_{2} \mathrm{O}_{2}$ treatment. ${ }^{*} p<0.05,{ }^{* *} p<0.01$, and ${ }^{* * *} p<0.001$. Ctl: control; NC: negative control. 
$\mathrm{NC}$

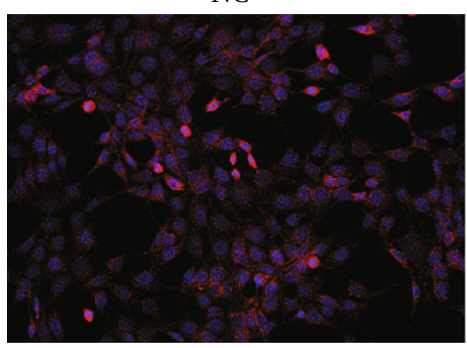

si-AW112010

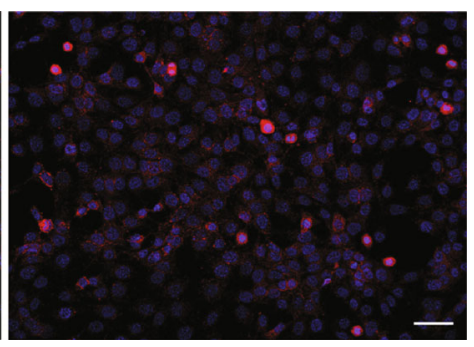

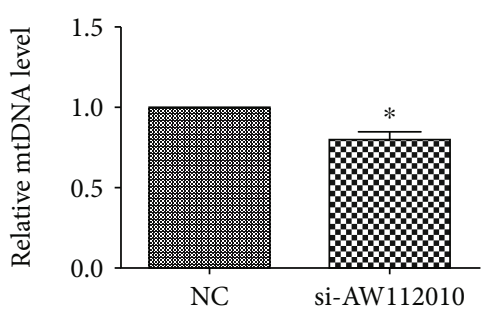

(b)
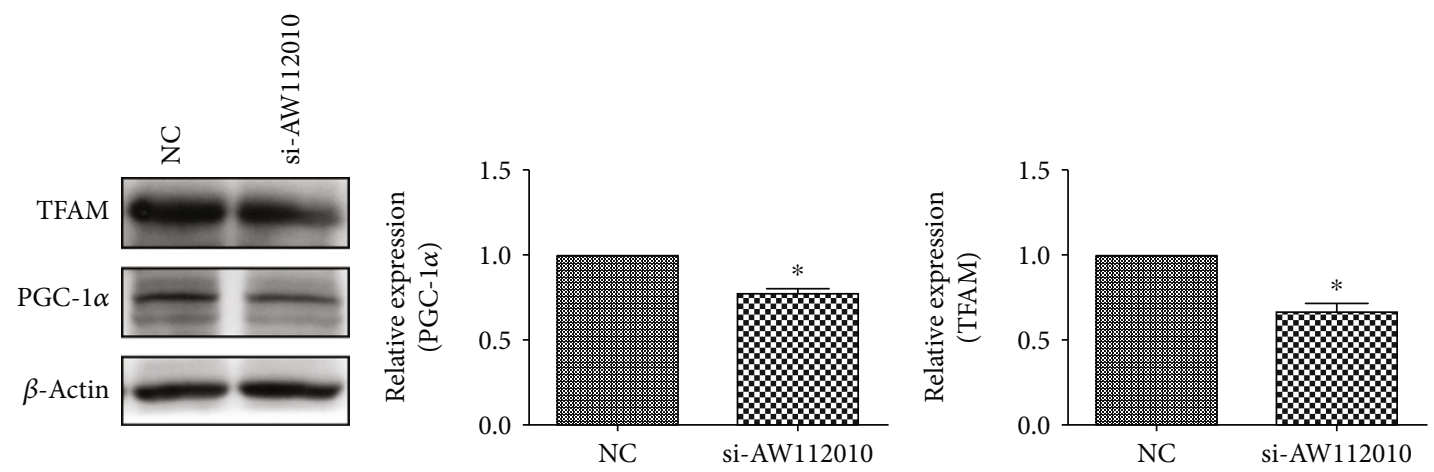

(c)
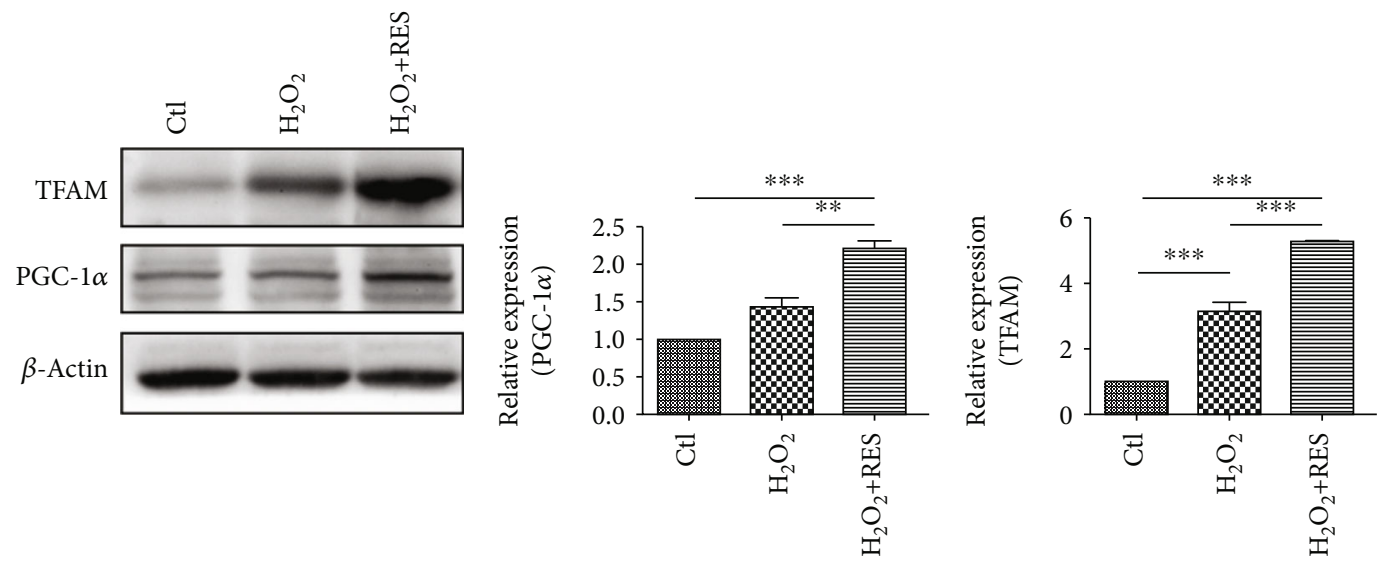

(d)

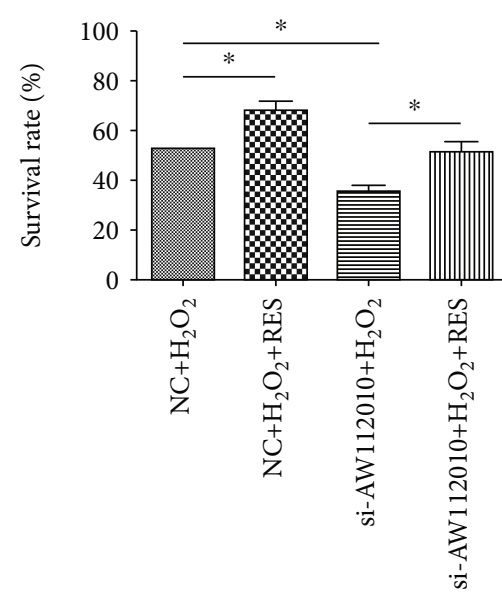

(e)

FIgUre 4: Continued. 

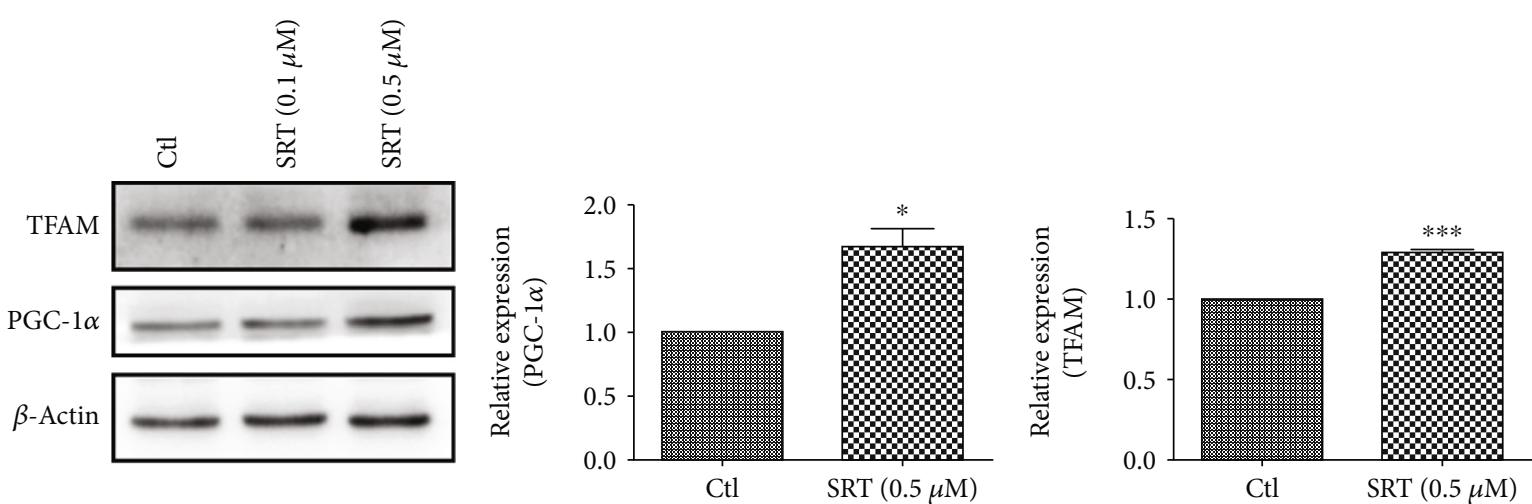

(f)

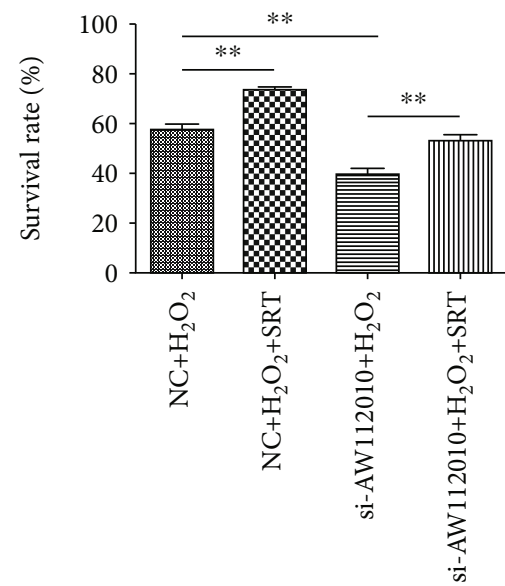

(g)

FIgURE 4: AW112010 induced mitochondrial biogenesis in HEI-OC1 cells. (a) Mitochondrial mass was measured using MitoTracker Red CMXRos probes. Mitochondrial staining showed reduced red fluorescence in AW112010 knockdown cells. Scale bar: $50 \mu \mathrm{m}$. (b) mtDNA (D-loop) levels were detected by qPCR and normalized to nuclear gene beta2-microglobulin. Silencing of AW112010 reduced the mtDNA content in HEI-OC1 cells. (c) Western blot and densitometry of mitochondrial biogenesis-related transcription factors PGC- $1 \alpha$ and TFAM. Levels of PGC- $1 \alpha$ and TFAM were normalized to the $\beta$-actin level. AW 112010 knockdown reduced the expression of PGC-1 $\alpha$ and TFAM in HEI-OC1 cells. (d) HEI-OC1 cells were preincubated with resveratrol $(5 \mu \mathrm{M})$ for $24 \mathrm{~h}$ and then treated with $\mathrm{H}_{2} \mathrm{O}_{2}(1 \mathrm{mM})$ for $6 \mathrm{~h}$. Western blot showed that resveratrol activated the expression of PGC-1 $\alpha$ and TFAM in HEI-OC1 cells. (e) After AW112010 Smart Silencer transfection, cells were preincubated with resveratrol $(5 \mu \mathrm{M})$ for $24 \mathrm{~h}$ and then treated with $\mathrm{H}_{2} \mathrm{O}_{2}(1 \mathrm{mM})$ for $2 \mathrm{~h}$. CCK-8 assay revealed that resveratrol elevated cell viability against $\mathrm{H}_{2} \mathrm{O}_{2}$ insults in HEI-OC1 cells. (f) HEI-OC1 cells were incubated with 0.1 or $0.5 \mu \mathrm{M}$ STR1720 for $24 \mathrm{~h}$. Western blot showed that $0.5 \mu \mathrm{M}$ SRT1720 induced the expression of PGC-1 $\alpha$ and TFAM in HEI-OC1 cells. (g) After AW112010 Smart Silencer transfection, cells were preincubated with SRT1720 $(0.5 \mu \mathrm{M})$ for $24 \mathrm{~h}$ and then treated with $\mathrm{H}_{2} \mathrm{O}_{2}\left(1 \mathrm{mM}^{2}\right)$ for $2 \mathrm{~h}$. CCK-8 assay revealed that SRT1720 increased cell viability against $\mathrm{H}_{2} \mathrm{O}_{2}$ insults in HEI-OC1 cells. ${ }^{*} p<0.05,{ }^{* *} p<0.01$, and ${ }^{* * *} p<$ 0.001. RES: resveratrol; SRT: SRT1720.

AW112010 may be involved in oxidative stress and energy metabolism.

Given that the mitochondrion is the central organelle in regulating energy metabolism and oxidative stress, we wondered about the role of AW112010 in mitochondrial function. First, the expression of AW112010 was knocked down by transfection of Smart Silencer (a commercial mixture of siRNA and ASO) in HEI-OC1 cells. The knockdown efficiency was confirmed by RT-qPCR $(p<0.01$, Figure 3(d)). Then, we measured the ATP level, mitochondrial ROS generation, mitochondrial membrane potential (MMP), and cell viability in HEI-OC1 cells. AW112010 knockdown decreased the cellular ATP level $(p<0.05$, Figure 3(e)). The JC-1 assay showed that AW112010 inhibition decreased MMP $(p<0.05$, Figure 3(f)). AW112010 inhibition elevated mitochondrial ROS generation under $\mathrm{H}_{2} \mathrm{O}_{2}$ stimulation (Figure $3(\mathrm{~g})$ ). The CCK-8 assay revealed that AW112010 knockdown slightly decreased cell survival $(p<0.05$, Figure $3(\mathrm{~h}))$. Under $\mathrm{H}_{2} \mathrm{O}_{2}(1 \mathrm{mM})$ stimulation, AW112010 knockdown significantly reduced the cell survival rate compared to the control group $(p<0.01$, Figure $3(\mathrm{~h}))$. Furthermore, HEI-OC1 cells were transfected with pcDNA3.1-AW112010 plasmid to express AW112010 $(p<0.01$, Figure 3(i)). AW112010 overexpression increased cell survival in $\mathrm{H}_{2} \mathrm{O}_{2}$-stressed HEI-OC1 cells (Figure 3(j)). These results suggest that AW112010 promoted mitochondrial function and cell survival in HEI-OC1 cells. HEI-OC1 Cells. To determine whether AW112010 plays a 

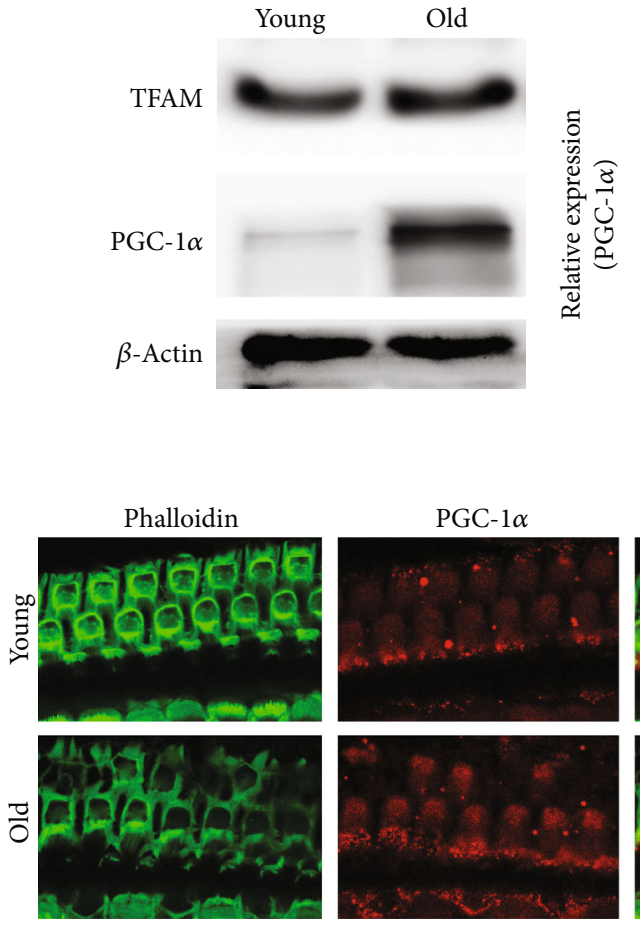

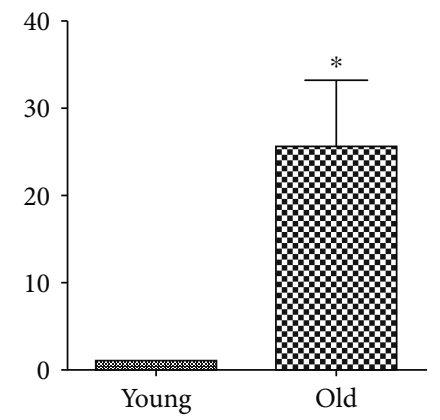

(a)

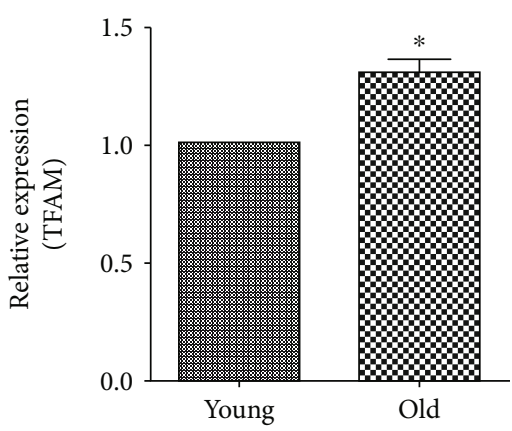

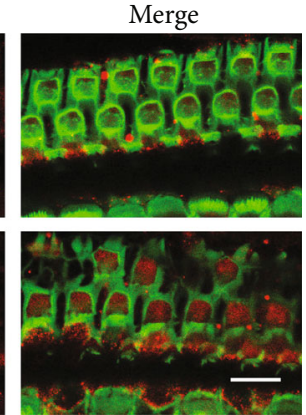

(b)
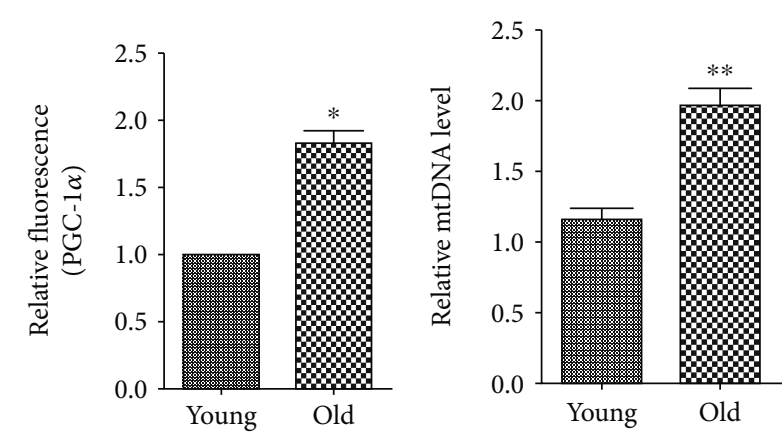

(c)

Figure 5: Activation of the mitochondrial biogenesis process in the cochlea of aged C57BL/6 mice. (a) Western blot and densitometry of PGC- $1 \alpha$ and TFAM. Levels of PGC- $1 \alpha$ and TFAM were normalized to the $\beta$-actin level. Increased expressions of PGC- $1 \alpha$ and TFAM were detected in the cochlea of aged mice. (b) Immunocytochemical analysis showed elevated immunolabeling of PGC-1 $\alpha$ in the outer hair cells of aged mice. Scale bar: $10 \mu \mathrm{m}$. (c) qPCR analysis showed an increased mtDNA level in aged cochlea tissues. ${ }^{*} p<0.05,{ }^{* *} p<0.01$.

role in mitochondrial biogenesis, the mitochondrial mass was assessed by mitochondrial probe staining. Silencing of AW112010 decreased the red fluorescence of mitochondrial staining in HEI-OC1 cells (Figure 4(a)). Further, the level of mtDNA (D-loop) was reduced in AW112010 silencing cells compared to the control $(p<0.05$, Figure $4(\mathrm{~b}))$. In addition, Western blot analysis showed that the levels of mitochondrial biogenesis transcription factors PGC- $1 \alpha$ and TFAM decreased in AW112010 silencing cells $(p<0.05$, Figure 4(c)). Resveratrol and SRT1720 have been proposed to activate mitochondrial biogenesis. Resveratrol $(5 \mu \mathrm{M})$ and SRT1720 $(0.5 \mu \mathrm{M})$ evidently increased the protein level of PGC- $1 \alpha$ and TFAM in HEI-OC1 cells (Figures 4(d) and $4(\mathrm{f}))$. Treatment with resveratrol $(5 \mu \mathrm{M})$ and SRT1720 $(0.5 \mu \mathrm{M})$ protected HEI-OC1 cells against $\mathrm{H}_{2} \mathrm{O}_{2}$ insults (Figures 4(e) and 4(g)). These data indicate that AW112010 activated mitochondrial biogenesis in HEI-OC1 cells.

3.5. Activated Mitochondrial Biogenesis Process in the Cochlea of Aged C57BL/6 Mice. To determine the status of the mitochondrial biogenesis process in the aged cochlea, cochlear tissues from young and aged C57BL/6 mice were collected for detection of mitochondrial biogenesis-related transcription factors. Western blot analysis showed that the levels of PGC- $1 \alpha$ and TFAM were significantly elevated in aged cochlea samples $(p<0.05$, Figure 5(a)). Immunocytochemical analysis further showed that the PGC-1 $\alpha$ immunolabeling was increased in the outer hair cells of aged cochlea samples $(p<0.05$, Figure 5(b)). Moreover, the mtDNA level was higher in aged cochlea tissues than in the young $(p<0.01$, Figure 5(c)). Taken together, these findings suggest that the mitochondrial biogenesis process was activated in the cochlea of aged C57BL/6 mice.

3.6. AW112010 Modulates the Activation of AMPK in HEIOC1 Cells. AMPK functions as a cellular energy sensor and is a critical mediator of mitochondrial biogenesis [11]. Inhibition of AMPK by Compound $\mathrm{C}$ decreased the expression of mitochondrial biogenesis transcription factor PGC- $1 \alpha$ $(p<0.05$, Figure 6(a)). To test if AW112010 may regulate mitochondrial biogenesis via the AMPK pathway, the expression of activated AMPK was detected by Western blot assays. AW112010 silencing reduced the level of phosphorylated AMPK $(p<0.01$, Figure 6(b)), while AW112010 overexpression increased it $(p<0.05$, Figure 6(c)). These data suggest that AW112010 positively modulated AMPK activation in HEI-OC1 cells.

3.7. Transcription Factor Arid5b Regulates AW112010 Expression. We next explored the mechanism of upregulation of AW112010 in aged cochlea samples. Using the QIAGEN database (http://www.sabiosciences.com/chipqpc rsearch.php), we found that Arid5b is the top one transcription factor for AW112010. Further, siRNA-mediated knockdown of Arid5b was performed in HEI-OC1 cells (Figure 7(f)). Arid5b knockdown cells showed a marked 


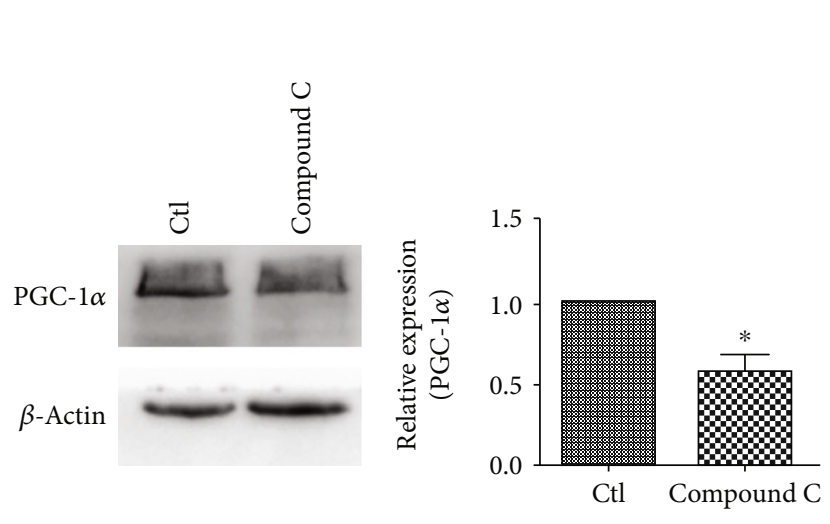

(a)

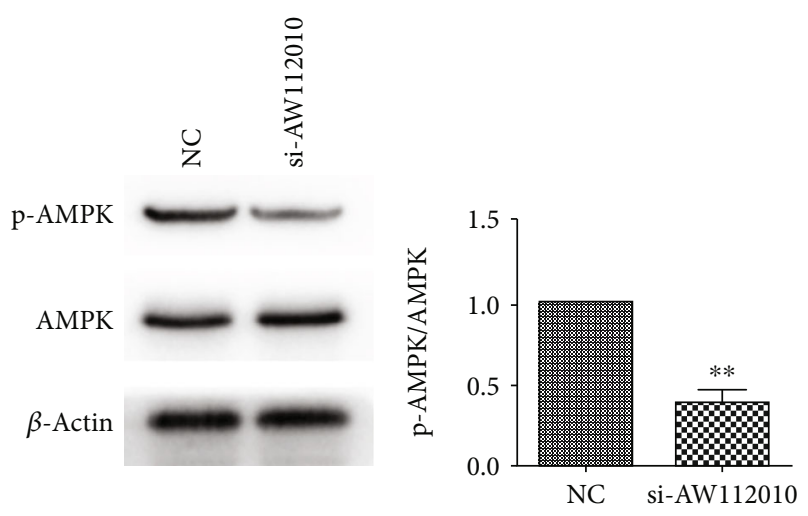

(b)

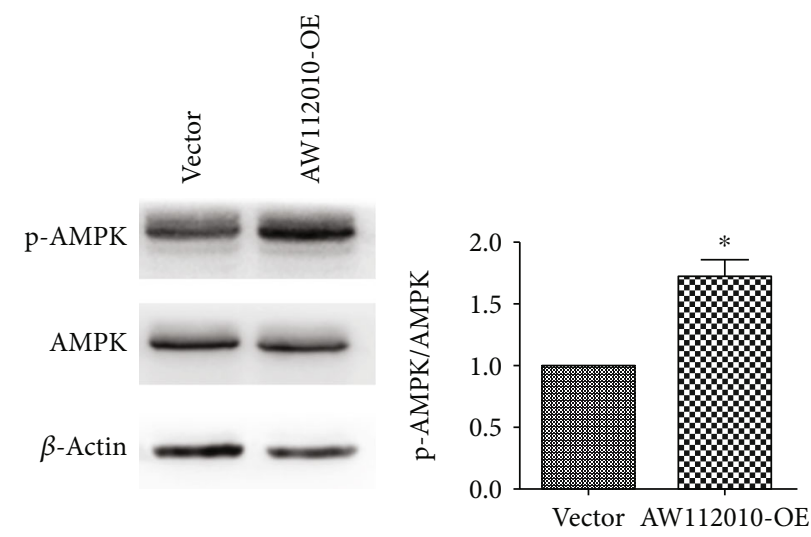

(c)

FIgURE 6: AW112010 induced the activation of AMPK in HEI-OC1 cells. (a) Western blot assay showed that AMPK inhibitor Compound C decreased the expression of PGC- $1 \alpha$ in HEI-OC1 cells. (b) Western blot assay revealed that AW112010 knockdown reduced the expression of phosphorylated AMPK (p-AMPK) in HEI-OC1 cells. (c) Western blot assay showed that AW112010 overexpression increased the expression of p-AMPK in HEI-OC1 cells. ${ }^{*} p<0.05,{ }^{* *} p<0.01$.

decrease in AW112010 expression $(p<0.05$, Figure $7(\mathrm{a}))$. Moreover, the expression of Arid5b in the aged cochlea was examined. Similar to elevated AW112010, the Arid5b RNA and protein levels were increased in aged cochlea samples $(p<0.01$, Figures $7(\mathrm{~b})$ and $7(\mathrm{c})) . \mathrm{H}_{2} \mathrm{O}_{2}$ stimulation and serum deprivation induced Arid5b expression in HEI-OC1 cells $(p<0.01$, Figures $7(\mathrm{~d})$ and $7(\mathrm{e}))$. Additionally, Arid5b inhibition reduced the protein levels of PGC- $1 \alpha$ and TFAM $(p<0.05$, Figure $7(\mathrm{f}))$ and decreased the cell viability in HEI-OC1 cells (Figure $7(\mathrm{~g})$ ). These results indicate that transcription factor Arid5b positively regulated AW112010 expression and mitochondrial biogenesis.

\section{Discussion}

In this study, we identified dysregulated lncRNAs in the cochlea of aged C57BL/6 mice, which displayed early-onset AHL. We focused on the significantly upregulated AW112010 and its role in mitochondrial biogenesis. In HEIOC1 cells, AW112010 promoted mitochondrial function and mitochondrial biogenesis. In accordance with elevated AW112010 expression, the mitochondrial biogenesis process was activated in the aged cochlea. Moreover, AW112010 positively regulated the energy-sensitive AMPK signaling in HEI-OC1 cells. In addition, transcription factor Arid5b elevated in the aged cochlea and positively modulated the AW112010 level and mitochondrial biogenesis in HEI-OC1 cells (Figure 8).

Increasing evidence has demonstrated that lncRNAs play critical roles in aging-associated diseases [8, 15-17]. In this study, to the best of our knowledge, we first revealed the lncRNA profile in the aged cochlea in an AHL mouse model. These dysregulated lncRNAs may have favorable or harmful effects on the survival of cochlear hair cells. Among these altered lncRNAs, AW112010 was significantly increased in the aged cochlea. It has been reported to be induced following virus infection in microglia and astrocytes [14], but its function has not been characterized. Cells usually suffer from excessive oxidative stress and energy shortage with aging [18]. We speculated that AW112010 might be induced under such adverse situations in vitro. As expected, $\mathrm{H}_{2} \mathrm{O}_{2}$ stimulation and serum deprivation significantly triggered AW112010 expression in HEI-OC1 cells. In addition, AMPK inhibitor Compound C markedly reduced AW112010 expression, suggesting that AW112010 is sensitive to AMPK signaling which plays a key role in energy metabolism. These findings suggest that AW112010 may be involved in oxidative stress and energy metabolism.

Mitochondria are the cellular center for energy production as well as the major source of $\operatorname{ROS}[19,20]$. 


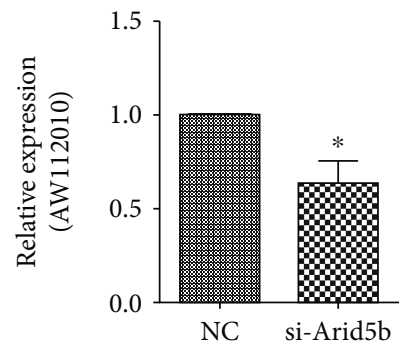

(a)

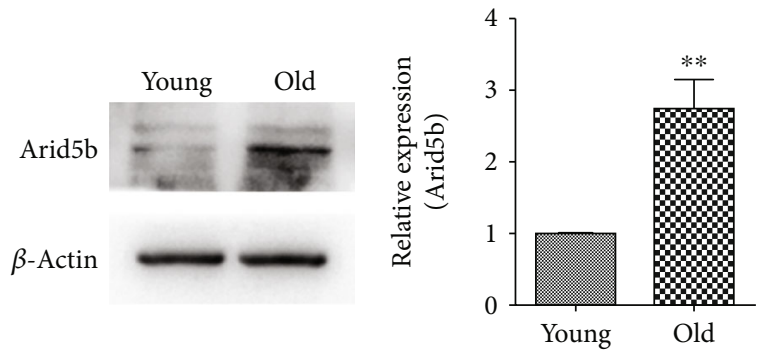

(c)
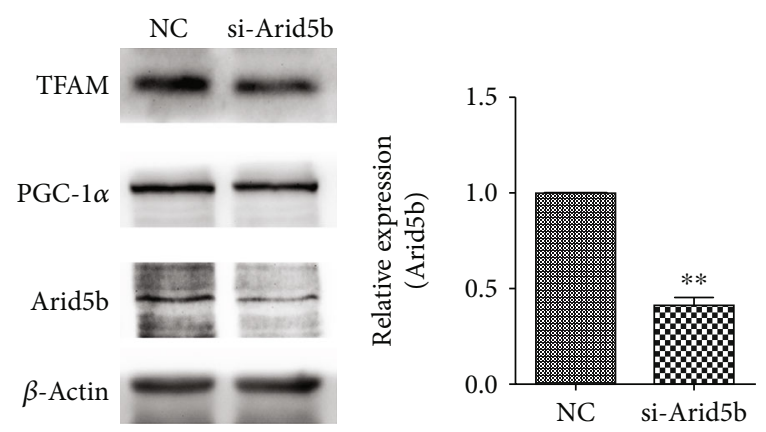

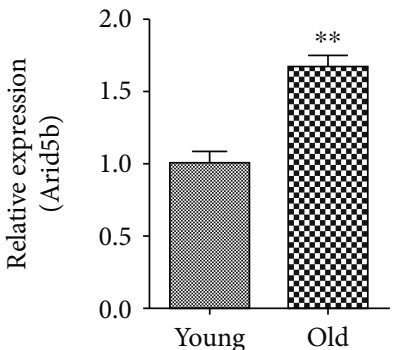

(b)

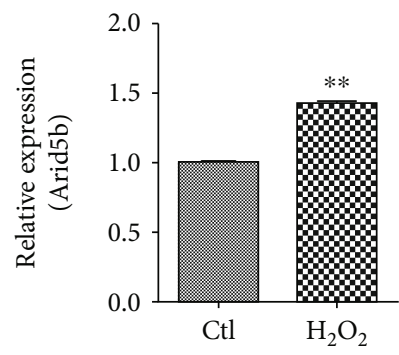

(d)
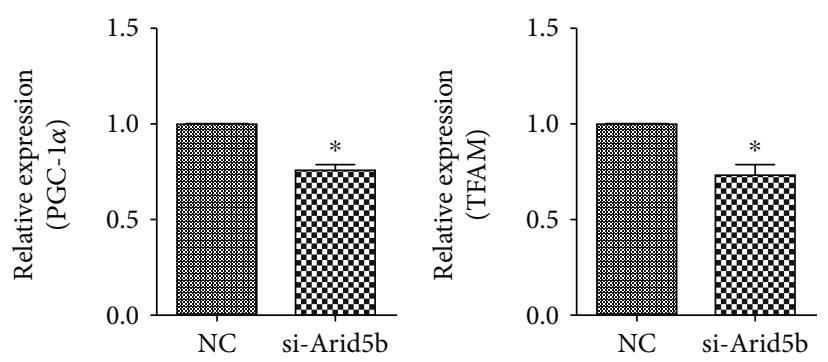

(f)

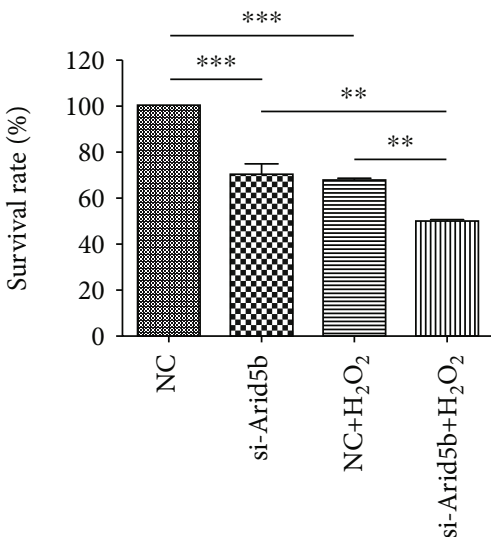

(g)

Figure 7: Transcription factor Arid5b modulated the expression of AW112010 and mitochondrial biogenesis process. (a) RT-qPCR assay revealed a reduced expression of AW112010 in Arid5b knockdown cells compared to negative control cells. The expression of Arid5b in the mouse cochlea was measured by RT-qPCR (b) and Western blot assay (c). Elevated mRNA and protein expression of Arid5b was detected in old mice compared to young mice. RT-qPCR assay showed an increased expression of Arid5b in HEI-OC1 cells under the treatment of $\mathrm{H}_{2} \mathrm{O}_{2}$ (d) and serum deprivation (e) in HEI-OC1 cells. (f) Western blot assay showed that Arid5b knockdown inhibited the expression of PGC- $1 \alpha$ and TFAM in HEI-OC1 cells. (g) CCK- 8 assay revealed decreased cell viability in Arid5b knockdown cells in the presence or absence of $1 \mathrm{mM} \mathrm{H}_{2} \mathrm{O}_{2}$ treatment compared to negative control cells. ${ }^{*} p<0.05,{ }^{* *} p<0.01$, and ${ }^{* * *} p<0.001$. 


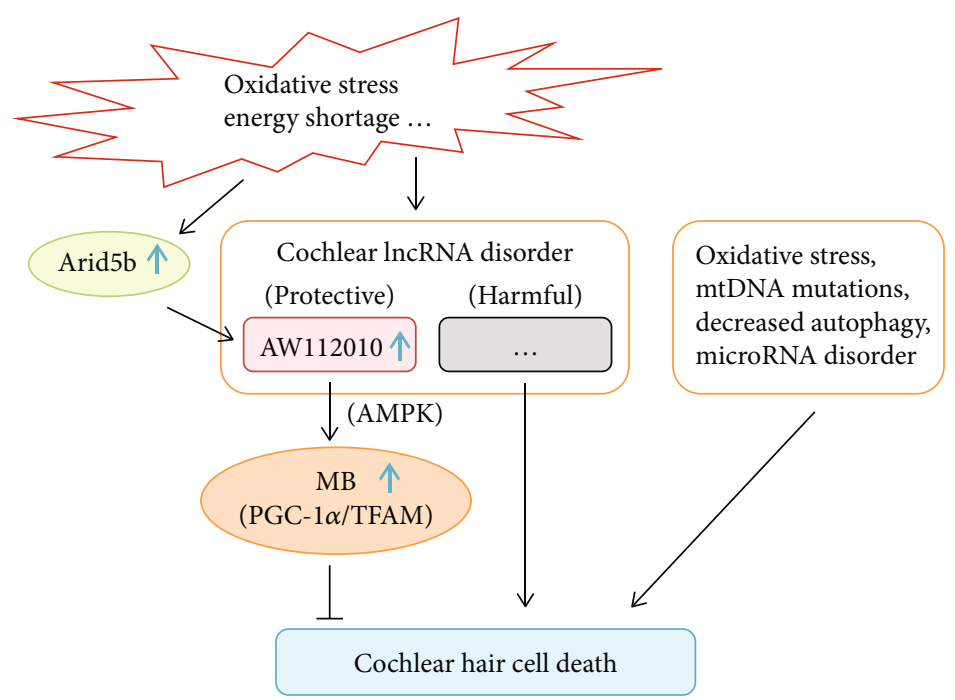

FIGURE 8: Schematic model demonstrating that activated Arid5b/AW112010 signaling promotes mitochondrial biogenesis in the cochlea. Oxidative stress, mitochondrial DNA (mtDNA) mutations, decreased autophagy, and microRNA disorder account for the death of cochlear hair cells $[1,2,4,5]$. During aging, adverse factors such as oxidative stress and energy shortage result in the dysregulation of cochlear lncRNAs, which might have protective or harmful roles in cochlear cell survival. In these adverse conditions, transcription factor Arid5b is elevated and activates the expression of AW112010. AW112010, probably via the activation of AMPK, increases the expression of PGC- $1 \alpha$ and TFAM and promotes mitochondrial biogenesis (MB) in the purpose of protecting the cochlear hair cell from death.

Mitochondrial dysfunction is an important mechanism underlying AHL development [1]. In this study, we revealed that AW112010 has a protective role in maintaining mitochondrial function in HEI-OC1 cells. Elevated AW112010 in the aged cochlea was probably a result of adaptive mechanism to cope with the shortage of energy and insult of ROS. A progressive increase in mitochondrial mass was observed during aging in multiple cell types of diverse organisms [11,21]. In aged tissues, cells usually suffer from energy shortage and excessive ROS. Mitochondrial biogenesis takes place under basal condition and is an adaptive response induced by cells to maintain energy demands [22]. In the present study, we observed an activated expression of PGC- $1 \alpha$ and TFAM in aged mouse cochlea. PGC- $1 \alpha$ is a core regulator of mitochondrial biogenesis $[23,24]$. A recent study revealed that IncRNA Tug1 regulated mitochondrial bioenergetics through transcriptionally activating PGC- $1 \alpha$ expression [13]. In this study, we demonstrated that AW112010 positively regulates mitochondrial mass and the expression of PGC- $1 \alpha$ and TFAM in HEI-OC1 cells. It is reasonable to speculate that an elevated AW112010 level in the aged cochlea must, at least partially, account for the activated mitochondrial biogenesis process in the purpose of maintaining mitochondrial function. Resveratrol and SRT1720 have been demonstrated to promote mitochondrial biogenesis via activation of sirtuin $1 /$ PGC- $1 \alpha$ signaling $[25,26]$. In our study, resveratrol and SRT1720 induced the expression of PGC- $1 \alpha$ and TFAM and rescued cell viability under oxidative stress in AW112010 silencing cells. Activation of PGC- $1 \alpha$ by genetic or pharmacological methods rescued the phenotypes of genetic models of Parkinson's disease [27]. Our study and others' together supported the notion that promotion of mitochondrial biogenesis may serve as an effective method to treating aging-related diseases.

Mitochondrial biogenesis is controlled by multiple signaling pathways like AMPK, which is the major energy sensor in cells $[11,28]$. AMPK phosphorylates PGC- $1 \alpha$ and induces mitochondrial biogenesis [11]. In this study, we observed that AW112010 induced the activation of AMPK. AW112010 might regulate mitochondrial biogenesis through AMPK signaling. To explore the upstream regulator of AW112010, we found that transcription factor Arid5b mediated the expression of AW112010 in HEI-OC1 cells. In addition, similar to the AW112010 expression pattern, the level of Arid5b was elevated in aged cochlea samples. Arid5b might be partially responsible for the overexpression of AW112010 in the aged cochlea. In addition, Arid5b positively modulated the expression of PGC- $1 \alpha$ and TFAM. Under oxidative stress and energy shortage conditions, the Arid5b/AW112010 signaling might be activated and attempted to recover cellular homeostasis in the cochlea of aged mice. Arid5b has also been proposed to be an important mediator in lipid metabolism via inducing the expression of CCAA T/enhancer-binding protein $\alpha(\mathrm{C} / \mathrm{EBP} \alpha)$ and peroxisome proliferator-activated receptor- $\gamma(\operatorname{PPAR} \gamma)$ in adipocytes $[29,30]$.

In sum, our findings, for the first time, demonstrated that lncRNAs were dysregulated with aging in the cochlea of C57BL/6 mice. The Arid5b/AW112010 signaling was activated in aged mouse cochlea and positively modulated the mitochondrial biogenesis process to maintain mitochondrial function. Our results increase our understanding of lncRNA-mediated mitochondrial biogenesis in maintaining hair cell homeostasis and provide potential targets for developing lncRNA-based therapeutics in AHL. 


\section{Data Availability}

The sequencing data have been submitted to the NCBI Gene Expression Omnibus (GEO) under accession number GSE127204.

\section{Conflicts of Interest}

The authors declare no conflicts of interest.

\section{Acknowledgments}

This work was supported by grants from the National Natural Science Foundation of China (81771018, 81570916, and 81570935).

\section{References}

[1] T. Yamasoba, F. R. Lin, S. Someya, A. Kashio, T. Sakamoto, and K. Kondo, "Current concepts in age-related hearing loss: epidemiology and mechanistic pathways," Hearing Research, vol. 303, pp. 30-38, 2013.

[2] E. Tavanai and G. Mohammadkhani, "Role of antioxidants in prevention of age-related hearing loss: a review of literature," European Archives of Oto-Rhino-Laryngology, vol. 274, no. 4, pp. 1821-1834, 2017.

[3] S. Someya, J. Xu, K. Kondo et al., "Age-related hearing loss in C57BL/6J mice is mediated by Bak-dependent mitochondrial apoptosis," Proceedings of the National Academy of Sciences of the United States of America, vol. 106, no. 46, pp. 1943219437, 2009.

[4] H. Xiong, J. Pang, H. Yang et al., "Activation of miR34a/SIRT1/p53 signaling contributes to cochlear hair cell apoptosis: implications for age-related hearing loss," Neurobiology of Aging, vol. 36, no. 4, pp. 1692-1701, 2015.

[5] J. Pang, H. Xiong, P. Lin et al., "Activation of miR-34a impairs autophagic flux and promotes cochlear cell death via repressing ATG9A: implications for age-related hearing loss," Cell Death \& Disease, vol. 8, no. 10, article e3079, 2017.

[6] J. J. Quinn and H. Y. Chang, "Unique features of long noncoding RNA biogenesis and function," Nature Reviews Genetics, vol. 17, no. 1, pp. 47-62, 2016.

[7] K. C. Wang and H. Y. Chang, "Molecular mechanisms of long noncoding RNAs," Molecular Cell, vol. 43, no. 6, pp. 904-914, 2011.

[8] M. Esteller, "Non-coding RNAs in human disease," Nature Reviews Genetics, vol. 12, no. 12, pp. 861-874, 2011.

[9] K. Ushakov, T. Koffler-Brill, A. Rom, K. Perl, I. Ulitsky, and K. B. Avraham, "Genome-wide identification and expression profiling of long non-coding RNAs in auditory and vestibular systems," Scientific Reports, vol. 7, no. 1, article 8637, 2017.

[10] B. Wang, E. Ding, H. Shen et al., "Association of TagSNP in lncRNA HOTAIR with susceptibility to noise-induced hearing loss in a Chinese population," Hearing Research, vol. 347, pp. 41-46, 2017.

[11] K. Palikaras and N. Tavernarakis, "Mitochondrial homeostasis: the interplay between mitophagy and mitochondrial biogenesis," Experimental Gerontology, vol. 56, pp. 182-188, 2014.
[12] C. Ploumi, I. Daskalaki, and N. Tavernarakis, "Mitochondrial biogenesis and clearance: a balancing act," The FEBS Journal, vol. 284, no. 2, pp. 183-195, 2017.

[13] J. Long, S. S. Badal, Z. Ye et al., "Long noncoding RNA Tug1 regulates mitochondrial bioenergetics in diabetic nephropathy," The Journal of Clinical Investigation, vol. 126, no. 11, pp. 4205-4218, 2016.

[14] S. Madeddu, T. A. Woods, P. Mukherjee, D. Sturdevant, N. B. Butchi, and K. E. Peterson, "Identification of glial activation markers by comparison of transcriptome changes between astrocytes and microglia following innate immune stimulation," PLoS One, vol. 10, no. 7, article e127336, 2015.

[15] D. Pereira Fernandes, M. Bitar, F. Jacobs, and G. Barry, "Long non-coding RNAs in neuronal aging," Non-Coding RNA, vol. 4, no. 2, p. 12, 2018.

[16] J. S. Sunwoo, S. T. Lee, W. Im et al., "Altered expression of the long noncoding RNA NEAT1 in Huntington's disease," Molecular Neurobiology, vol. 54, no. 2, pp. 1577-1586, 2017.

[17] Y. Ruan, N. Lin, Q. Ma et al., "Circulating LncRNAs analysis in patients with type 2 diabetes reveals novel genes influencing glucose metabolism and islet $\beta$-cell function," Cellular Physiology and Biochemistry, vol. 46, no. 1, pp. 335-350, 2018.

[18] R. Uzhachenko, K. Boyd, D. Olivares-Villagomez et al., "Mitochondrial protein Fus1/Tusc2 in premature aging and age-related pathologies: critical roles of calcium and energy homeostasis," Aging, vol. 9, no. 3, pp. 627-649, 2017.

[19] M. F. Beal, "Mitochondria and neurodegeneration," in Mitochondrial Disorders, Springer, Paris, 2007.

[20] A. T. Hoye, J. E. Davoren, P. Wipf, M. P. Fink, and V. E. Kagan, "Targeting mitochondria," Accounts of Chemical Research, vol. 41, no. 1, pp. 87-97, 2008.

[21] K. Palikaras, E. Lionaki, and N. Tavernarakis, "Coupling mitogenesis and mitophagy for longevity," Autophagy, vol. 11, no. 8, pp. 1428-1430, 2015.

[22] J. A. Funk, S. Odejinmi, and R. G. Schnellmann, "SRT1720 induces mitochondrial biogenesis and rescues mitochondrial function after oxidant injury in renal proximal tubule cells," The Journal of Pharmacology and Experimental Therapeutics, vol. 333, no. 2, pp. 593-601, 2010.

[23] K. Hasegawa, T. Yasuda, C. Shiraishi et al., "Promotion of mitochondrial biogenesis by necdin protects neurons against mitochondrial insults," Nature Communications, vol. 7, no. 1, article 10943, 2016.

[24] J. Viña, M. C. Gomez-Cabrera, C. Borras et al., "Mitochondrial biogenesis in exercise and in ageing," Advanced Drug Delivery Reviews, vol. 61, no. 14, pp. 1369-1374, 2009.

[25] A. Ferretta, A. Gaballo, P. Tanzarella et al., "Effect of resveratrol on mitochondrial function: implications in parkinassociated familiar Parkinson's disease," Biochimica et Biophysica Acta (BBA) - Molecular Basis of Disease, vol. 1842, no. 7, pp. 902-915, 2014.

[26] R. M. Whitaker, D. Corum, C. C. Beeson, and R. G. Schnellmann, "Mitochondrial biogenesis as a pharmacological target: a new approach to acute and chronic diseases," Annual Review of Pharmacology and Toxicology, vol. 56, no. 1, pp. 229-249, 2016.

[27] C. H. Ng, A. H. Basil, L. Hang et al., "Genetic or pharmacological activation of the Drosophila PGC- $1 \alpha$ ortholog spargel rescues the disease phenotypes of genetic models of Parkinson's disease," Neurobiology of Aging, vol. 55, pp. 33-37, 2017. 
[28] C. Li, X. Y. Ding, D. M. Xiang et al., "Enhanced M1 and impaired M2 macrophage polarization and reduced mitochondrial biogenesis via inhibition of AMP kinase in chronic kidney disease," Cellular Physiology and Biochemistry, vol. 36, no. 1, pp. 358-372, 2015.

[29] T. Yamakawa, K. Sugimoto, R. H. Whitson, and K. Itakura, "Modulator recognition factor-2 regulates triglyceride metabolism in adipocytes," Biochemical and Biophysical Research Communications, vol. 391, no. 1, pp. 277-281, 2010.

[30] T. Yamakawa, R. H. Whitson, S.-L. Li, and K. Itakura, "Modulator recognition factor-2 is required for adipogenesis in mouse embryo fibroblasts and 3T3-L1 cells," Molecular Endocrinology, vol. 22, no. 2, pp. 441-453, 2008. 


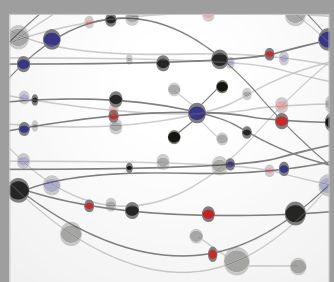

The Scientific World Journal
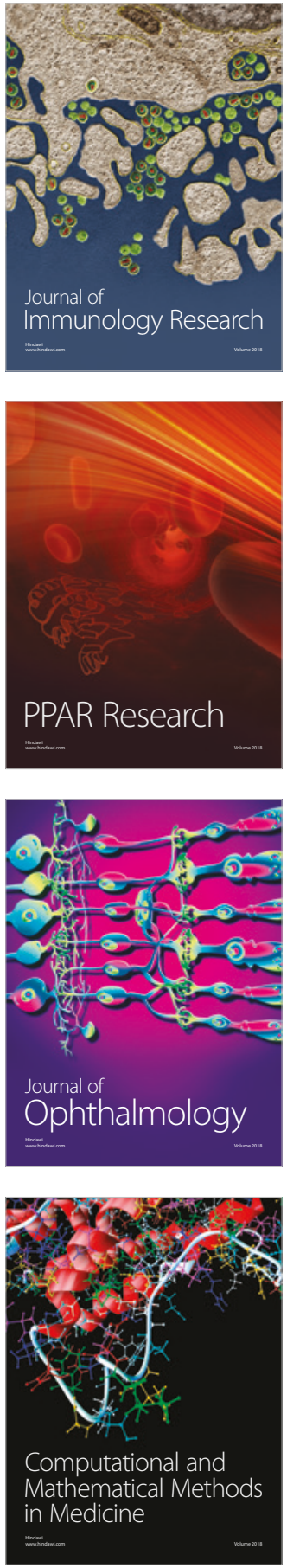

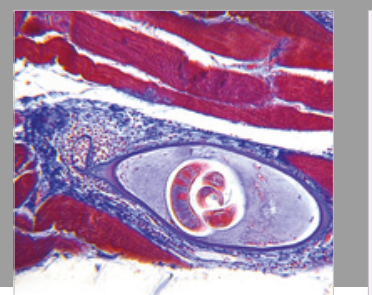

Gastroenterology Research and Practice

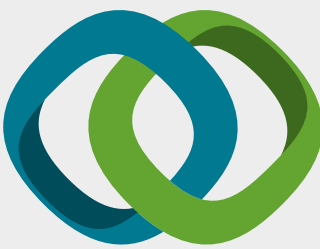

\section{Hindawi}

Submit your manuscripts at

www.hindawi.com
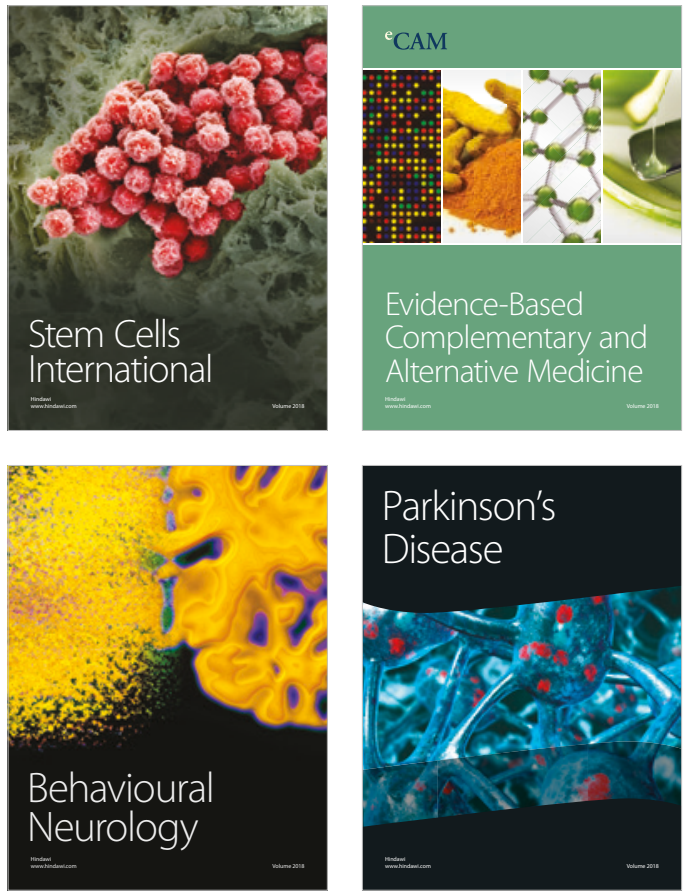

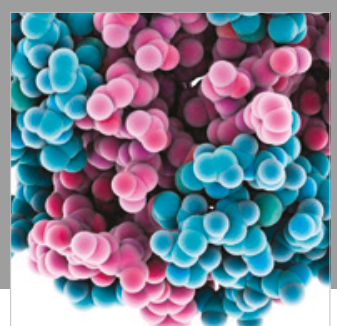

ournal of

Diabetes Research

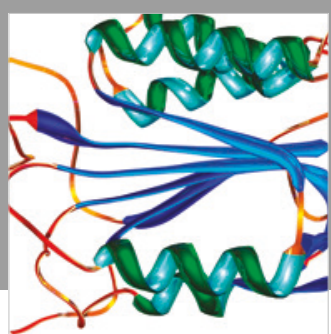

Disease Markers
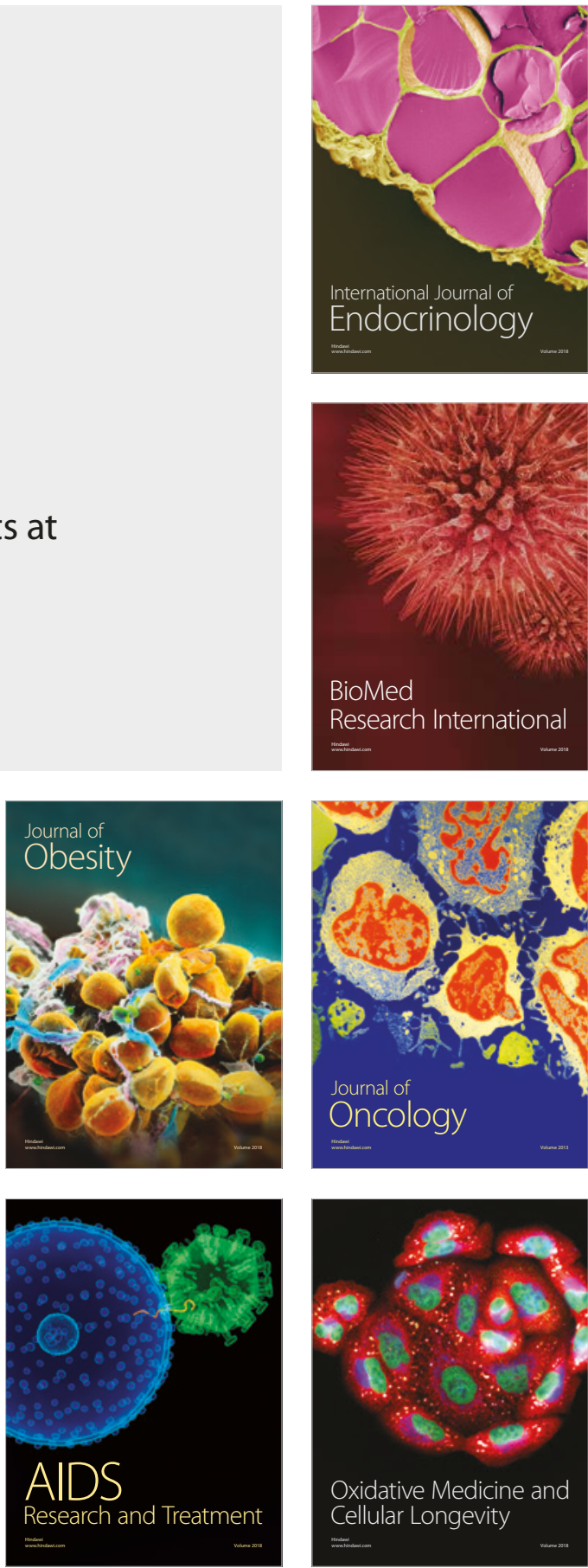